ESAIM: PROCEEDINGS AND SURVEYS, 2017, Vol. 57, p. 64-69

Oana Silvia Serea \& Walter Briec Editors

\title{
EQUILIBRIA FOR GAMES IN IDEMPOTENT MEASURES
}

\author{
TARAS RADUL ${ }^{1,2}$
}

\begin{abstract}
This paper is devoted to non-cooperative games in idempotent measures. We consider two kinds of such games. Firstly, players are allowed to play their mixed idempotent strategies. We also consider games where belief of each player about a choice of the strategy by the other players is an idempotent measure. We prove existence of equilibria in both cases and discuss the difference between them.
\end{abstract}

\section{INTRODUCTION}

The classical Nash equilibrium theory is based on fixed point theory and was developed in frames of linear convexity. The mixed strategies of a player are probability (additive) measures on a set of pure strategies. But an interest to Nash equilibria in more general frames is rapidly growing in last decades. For instance, Aliprantis, Florenzano and Tourky [1] work in ordered topological vector spaces, Luo [9] in topological semilattices, Vives [17] in complete lattices. Briec and Horvath [2] proved the existence of Nash equilibrium point for $B$-convexity and Max-Plus convexity.

We can use additive measures only when we know precisely probabilities of all events considered in a game. However, it is not a case in many modern economic models. The decision theory under uncertainty considers a model when probabilities of states are either not known or imprecisely specified. Gilboa [6] and Schmeidler [14] axiomatized expectations expressed by Choquet integrals attached to non-additive measures called capacities (fuzzy measures), as a formal approach to decision-making under uncertainty.

Dow and Werlang [4] used this approach for two players game where belief of each player about a choice of the strategy by the other player is a capacity. They introduced some equilibrium notion for such games and proved its existence. This result was extended onto games with arbitrary finite number of players in [5]. The same approach was considered in [11], but expected payoff function was defined by Sugeno integral.

Kozhan and Zarichnyi introduced in [8] an alternative to Dow and Werlang's concept of Nash equilibrium of a game where players are allowed to form non-additive beliefs about opponent's decision but also to play their mixed non-additive strategies. Such games were called by the authors as games in capacities. The expected payoff function was there defined using a Choquet integral. This concept was also considered by Glycopantis and Muir [7]. The notion of Nash equilibrium of a game with expected payoff function defined using Sugeno integral was considered in [12].

In this paper we apply both concepts of equilibria for games in idempotent (Maslov) measures. Let us remark that Max-Plus convexity considered in [2] is related to idempotent measures in the same sense as linear convexity is related to probability measures (see [13] and [15] for more details). We prove in Section 2 Nash equilibrium theorem for Max-Plus convexity in general case (Briec and Horvath proved in [2] the existence

\footnotetext{
1 Institute of Mathematics, Casimirus the Great University of Bydgoszcz, Poland;e-mail: tarasradul@yahoo.co.uk

2 Department of Mechanics and Mathematics, Ivan Franko National University of Lviv, Ukraine.
}

(C) EDP Sciences, SMAI 2017 
of Nash equilibrium for $B$-convex and Max-Plus convex finite-dimensional compacta). We use this result in Section 3 to prove the existence of Nash equilibrium for games in idempotent measures. In Section 4 we prove the existence of equilibrium in the sense of Dow and Verlang and discuss a difference between two concepts.

\section{IDEMPOTENT MEASURES: PRELIMINARIES}

In the sequel, all maps will be assumed to be continuous. Let $X$ be a compact Hausdorff space. We shall denote the Banach space of continuous functions on $X$ endowed with the sup-norm by $\mathrm{C}(\mathrm{X})$. For any $c \in R$ we shall denote the constant function on $X$ taking the value $c$ by $c_{X}$.

Let $\mathbb{R}_{\max }=\mathbb{R} \cup\{-\infty\}$ be the metric space endowed with the metric $\varrho$ defined by $\varrho(x, y)=\left|e^{x}-e^{y}\right|$. Following the notation of idempotent mathematics (see e.g., [10]) we use the notations $\oplus$ and $\odot$ in $\mathbb{R}$ as alternatives for max and + respectively. The convention $-\infty \odot x=-\infty$ allows us to extend $\odot$ and $\oplus$ over $\mathbb{R}_{\max }$.

Max-Plus convex sets were introduced in [20]. Let $\tau$ be a cardinal number. Given $x, y \in \mathbb{R}^{\tau}$ and $\lambda \in \mathbb{R}_{\max }$, we denote by $y \oplus x$ the coordinatewise maximum of $\mathrm{x}$ and $\mathrm{y}$ and by $\lambda \odot x$ the vector obtained from $x$ by adding $\lambda$ to each of its coordinates. A subset $A$ in $\mathbb{R}^{\tau}$ is said to be Max-Plus convex if $\alpha \odot a \oplus \beta \odot b \in A$ for all $a, b \in A$ and $\alpha, \beta \in \mathbb{R}_{\max }$ with $\alpha \oplus \beta=0$. In the following by Max-Plus convex compactum we mean a Max-Plus convex compact subset of $\mathbb{R}^{\tau}$.

We denote by $\odot: \mathbb{R} \times C(X) \rightarrow C(X)$ the map acting by $(\lambda, \varphi) \mapsto \lambda_{X}+\varphi$, and by $\oplus: C(X) \times C(X) \rightarrow C(X)$ the map acting by $(\psi, \varphi) \mapsto \max \{\psi, \varphi\}$.

Definition 1. [19] A functional $\mu: C(X) \rightarrow \mathbb{R}$ is called an idempotent measure (a Maslov measure) if

(1) $\mu\left(1_{X}\right)=1$;

(2) $\mu(\lambda \odot \varphi)=\lambda \odot \mu(\varphi)$ for each $\lambda \in \mathbb{R}$ and $\varphi \in C(X)$;

(3) $\mu(\psi \oplus \varphi)=\mu(\psi) \oplus \mu(\varphi)$ for each $\psi, \varphi \in C(X)$.

Let $I X$ denote the set of all idempotent measures on a compactum $X$. We consider $I X$ as a subspace of $\mathbb{R}^{C(X)}$. It is shown in [19] that $I X$ is a compact Max-Plus subset of $\mathbb{R}^{C(X)}$. The construction $I$ is functorial what means that for each continuous map $f: X \rightarrow Y$ we can consider a continuous map If $:$ IX IY defined as follows $\operatorname{If}(\mu)(\psi)=\mu(\psi \circ f)$ for $\mu \in I X$ and $\psi \in C(Y)$.

By $\delta_{x}$ we denote the Dirac measure supported by the point $x \in X$. It is shown in [19] that the set

$$
I_{\omega} X=\left\{\oplus_{i=1}^{n} \lambda_{i} \odot \delta_{x_{i}} \mid \lambda_{i} \in \mathbb{R}_{\max }, i \in\{1, \ldots, n\}, \oplus_{i=1}^{n} \lambda_{i}=0, x_{i} \in X, n \in \mathbb{N}\right\},
$$

(i.e., the set of idempotent measures of finite support) is dense in $I X$.

For a function $\psi \in C(X)$ we define a function $\pi_{\psi}: I X \rightarrow \mathbb{R}$ by the formula $\pi_{\psi}(\mu)=\mu(\psi)$ for $\mu \in I X$. It is easy to see that the function $\pi_{\psi}$ is continuous.

It is well known how to construct the tensor product of two (or finite number) probability measures. The tensor product for idempotent measures was described in [19]. More precisely, for each compacta $X_{1}, \ldots, X_{n}$ there exists a continuous map $\otimes: \prod_{i=1}^{n} I X_{i} \rightarrow I\left(\prod_{i=1}^{n} X_{i}\right)$ such that we have $I\left(p_{i}\right) \circ \otimes=\operatorname{pr}_{i}$ for each $i$ where $p_{i}: \prod_{j=1}^{n} X_{j} \rightarrow X_{i}$ and $\operatorname{pr}_{i}: \prod_{j=1}^{n} I X_{j} \rightarrow I X_{i}$ are the natural projections. If $\mu=\oplus_{i=1}^{n} \lambda_{i} \odot \delta_{x_{i}} \in I X$, $\nu=\oplus_{i=1}^{m} \beta_{i} \odot \delta_{y_{i}} \in I Y$, then

$$
\mu \otimes \nu=\oplus_{i=1}^{n} \oplus_{j=1}^{m}\left(\lambda_{i} \odot \beta_{j}\right) \odot \delta_{\left(x_{i}, y_{j}\right.} \in I(X \times Y)
$$

Analogous formula holds for any finite number of factors. Using density of $I_{\omega} X$ in $I X$ one can easily check the following lemma.

\section{Lemma 1.}

$$
\begin{gathered}
\nu_{1} \otimes \cdots \otimes \nu_{i-1} \otimes\left(\lambda_{1} \odot \mu_{1} \oplus \lambda_{2} \odot \mu_{2}\right) \otimes \nu_{i+1} \otimes \cdots \otimes \nu_{n}= \\
=\lambda_{1} \odot\left(\nu_{1} \otimes \cdots \otimes \nu_{i-1} \otimes \mu_{1} \otimes \nu_{i+1} \otimes \cdots \otimes \nu_{n}\right) \oplus \lambda_{2} \odot\left(\nu_{1} \otimes \cdots \otimes \nu_{i-1} \otimes \mu_{2} \otimes \nu_{i+1} \otimes \cdots \otimes \nu_{n}\right)
\end{gathered}
$$

for each $\nu_{j} \in I X_{j}$, where $j \neq i, \mu_{1}, \mu_{2} \in I X_{i}$ and $\lambda_{1}, \lambda_{2} \in \mathbb{R}_{\max }$ with $\lambda_{1} \oplus \lambda_{2}=0$. 


\section{NASh EQUilibrium FOR MAX-Plus CONVEXity}

A family $\mathcal{C}$ of closed subsets of a compactum $X$ is called a convexity on $X$ if $\mathcal{C}$ is stable for intersection and contains both $X$ and the empty set. The elements of $\mathcal{C}$ are called $\mathcal{C}$-convex (or simply convex).

A convexity $\mathcal{C}$ on $X$ is called $T_{4}$ (normal) if for each disjoint $C_{1}, C_{2} \in \mathcal{C}$ there exist $S_{1}, S_{2} \in \mathcal{C}$ such that $S_{1} \cup S_{2}=X, C_{1} \cap S_{2}=\emptyset$ and $C_{2} \cap S_{1}=\emptyset$.

Let us recall the definition of Nash equilibrium. We consider an $n$-players game $f: X=\prod_{i=1}^{n} X_{i} \rightarrow \mathbb{R}^{n}$ with compact Hausdorff spaces of strategies $X_{i}$. The coordinate function $f_{i}: X \rightarrow \mathbb{R}$ is called the payoff function of $i$-th player. For $x \in X$ and $t_{i} \in X_{i}$ we use the notation $\left(x ; t_{i}\right)=\left(x_{1}, \ldots, x_{i-1}, t_{i}, x_{i+1}, \ldots, x_{n}\right)$. A point $x \in X$ is called a Nash max-equilibrium (min-equilibrium) point if for each $i \in\{1, \ldots, n\}$ and for each $t_{i} \in X_{i}$ we have $f_{i}\left(x ; t_{i}\right) \leq f_{i}(x)\left(f_{i}\left(x ; t_{i}\right) \geq f_{i}(x)\right)$.

Now, let $\mathcal{C}_{i}$ be a convexity on $X_{i}$. We say that a function $f_{i}: X \rightarrow \mathbb{R}$ is quasiconcave (quasiconvex) with respect to the $i$-th variable if we have $\left(f_{i}^{x}\right)^{-1}([t ;+\infty)) \in \mathcal{C}_{i}\left(\left(f_{i}^{x}\right)^{-1}((-\infty ; t]) \in \mathcal{C}_{i}\right)$ for each $t \in \mathbb{R}$ and $x \in X$ where $f_{i}^{x}: X_{i} \rightarrow \mathbb{R}$ is the function defined as follows $f_{i}^{x}\left(t_{i}\right)=f_{i}\left(x ; t_{i}\right)$ for $t_{i} \in X_{i}$.

Theorem 1. [12] Let $f: X=\prod_{i=1}^{n} X_{i} \rightarrow \mathbb{R}^{n}$ be a game with a normal convexity $\mathcal{C}_{i}$ defined on each compactum $X_{i}$ such that all convex sets are connected, the function $f$ is continuous and the function $f_{i}: X \rightarrow \mathbb{R}$ is quasiconcave (quasiconvex) with respect to the $i$-th variable for each $i \in\{1, \ldots, n\}$. Then there exists a Nash max-equilibrium (min-equilibrium) point.

Let us remark that the previous theorem was proved in [11] only for max-equilibrium. But the proof is the same for min-equilibrium.

Now we apply these general concepts to Max-Plus convexity. The family $\mathcal{C}$ of the above definition will be the family of all closed Max-Plus convex subsets of a Max-Plus convex compactum. Evidently, each Max-Plus convex set is connected.

Lemma 2. Max-Plus convexity on a Max-Plus convex compactum $X \subset \mathbb{R}^{S}$ is normal.

Proof. Let $A$ and $B$ be two Max-Plus convex disjoint closed subsets of $X$. It follows from compactness of $A$ and $B$ and properties of product topology on $X \subset \mathbb{R}^{S}$ that there exists a finite subset $N$ of $S$ such that $p_{N}(A) \cap p_{N}(B)=\emptyset$ where $p_{N}: \mathbb{R}^{S} \rightarrow \mathbb{R}^{N}$ is the natural projection. Evidently $p_{N}(A)$ and $p_{N}(B)$ are Max-Plus convex disjoint compact subsets of $\mathbb{R}^{N}$. Theorem 7.1 from [3] and the fact that $\mathbb{B}$-convexity and Max-Plus convexity are isomorphic (see Section 5 of [2] for more details) imply that there exist two Max-Plus convex closed subsets $L_{1}, L_{2}$ of $\mathbb{R}^{N}$ such that $L_{1} \cup L_{2}=\mathbb{R}^{N}, L_{1} \cap p_{N}(B)=\emptyset=L_{2} \cap p_{N}(A)$. Then $\left(p_{N}\right)^{-1}\left(L_{1}\right) \cap X$ and $\left(p_{N}\right)^{-1}\left(L_{2}\right) \cap X$ are Max-Plus convex closed subsets of $X$ we are looking for.

Theorem 1 and Lemma 2 imply the following theorem.

Theorem 2. Let $f: X=\prod_{i=1}^{n} X_{i} \rightarrow \mathbb{R}^{n}$ be a game where each compactum $X_{i}$ is Max-Plus convex, the function $f$ is continuous and the function $f_{i}: X \rightarrow \mathbb{R}$ is Max-Plus quasiconcave (quasiconvex) with respect to the $i$-th variable for each $i \in\{1, \ldots, n\}$. Then there exists a Nash max-equilibrium (min-equilibrium) point.

\section{NASh EQUILIBRIUM IN MIXED STRATEGIES}

We consider a game $u: X=\prod_{i=1}^{n} X_{i} \rightarrow \mathbb{R}^{n}$ with compact Hausdorff spaces of pure strategies $X_{1}, \ldots, X_{n}$ and continuous payoff functions $u_{i}: \prod_{i=1}^{n} X_{i} \rightarrow \mathbb{R}$.

We define expected payoff functions $e u_{i}: I X_{1} \times \cdots \times I X_{n} \rightarrow \mathbb{R}$ by the formula $e u_{i}=\pi_{u_{i}} \circ \otimes$. Evidently, $e u_{i}$ are continuous. We discuss existence of Nash equilibrium in mixed strategies represented by idempotent measures. There exist a trivial solution of the problem for max-equilibrium. We can consider the natural order on $I X_{i}$. Then each $I X_{i}$ contains the greatest element $\mu_{i}$ defined by the formula $\mu_{i}(\psi)=\max \left\{\psi(x) \mid x \in X_{i}\right\}$ for $\psi \in C\left(X_{i}\right)$. Hence $\left(\mu_{1}, \ldots, \mu_{n}\right)$ is a Nash max-equilibrium. There is no such trivial solution for min-equilibrium, since $I X_{i}$ does not contain the smallest element.

The next lemma follows from the definition of the function $e u_{i}$ and Lemma 1. 
Lemma 3. The function $\mathrm{eu}_{i}$ is Max-Plus quasiconvex with respect to the $i$-th variable.

Now, Lemma 3 and Theorem 2 yield the following theorem.

Theorem 3. There exists a Nash min-equilibrium point for the game with the expected payoff functions eu : $I X_{1} \times \cdots \times I X_{n} \rightarrow \mathbb{R}$.

\section{Strategic EqUilibrium}

We consider in this section an equilibrium concept in the sense of [4] and [5] where players play pure strategies but belief of each player about a choice of the strategy by the other player is an idempotent measure.

Let $p: X=\prod_{i=1}^{n} X_{i} \rightarrow \mathbb{R}^{n}$ be an $n$-players game with compact Hausdorff spaces of strategies $X_{i}$. We assume that the function $p$ is continuous. For $i \in\{1, \ldots, n\}$ we denote by $X_{-i}=\prod_{j \neq i} X_{j}$ the set of strategy combinations which players other than $i$ could choose. For $x \in X$ the corresponding point in $X_{-i}$ we denote by $x_{-i}$. In contrast to standard game theory, beliefs of $i$-th player about opponents $\breve{S}$ behaviour are represented by idempotent measures on $X_{-i}$.

For $i \in\{1, \ldots, n\}$ we consider the expected payoff function $P_{i}: X_{i} \times I X_{-i} \rightarrow \mathbb{R}$ defined as follows $P_{i}\left(x_{i}, \nu\right)=$ $\nu\left(p_{i}^{x_{i}}\right)$, where the function $p_{i}^{x_{i}}: X_{-i} \rightarrow \mathbb{R}$ is defined by the formula $p_{i}^{x_{i}}\left(x_{-i}\right)=p_{i}\left(x_{i}, x_{-i}\right), x_{i} \in X_{i}$ and $\nu \in M X_{-i}$.

We are going to prove continuity of $P_{i}$.

Lemma 4. The map $P_{i}$ is continuous.

Proof. Consider any $x \in X_{i}, \nu \in I X_{-i}$ and put $P_{i}(x, \nu)=t \in \mathbb{R}$. Consider any $\varepsilon>0$. Since $X_{-i}$ is compact, there exists a neighborhood $O$ of $x$ such that

$$
\left|p_{i}(y, z)-p_{i}(x, z)\right|<\varepsilon / 2
$$

for each $y \in O$ and $z \in X_{-i}$.

Put $U=\left\{\mu \in I X_{-i}|| \mu\left(p_{i}^{x}\right)-\nu\left(p_{i}^{x}\right) \mid<\varepsilon / 2\right\}$. Then for each $(y, \mu) \in O \times U$ we have

$$
\left|P_{i}(x, \nu)-P_{i}(y, \mu)\right|=\left|\nu\left(p_{i}^{x}\right)-\mu\left(p_{i}^{y}\right)\right| \leq\left|\nu\left(p_{i}^{x}\right)-\mu\left(p_{i}^{x}\right)\right|+\left|\mu\left(p_{i}^{x}\right)-\mu\left(p_{i}^{y}\right)\right|<\varepsilon / 2+\varepsilon / 2=\varepsilon .
$$

Let us remark that the equality $\left|\mu\left(p_{i}^{x}\right)-\mu\left(p_{i}^{y}\right)\right| \leq \varepsilon / 2$ follows from the fact that each idempotent measure is non-expanding [19] and from the definition of the neighborhood $O$.

Hence the map $P_{i}$ is continuous and the lemma is proved.

For $\nu_{i} \in I\left(X_{-i}\right)$ denote by $R_{i}\left(\nu_{i}\right)=\left\{x \in X_{i} \mid P_{i}\left(x, \nu_{i}\right)=\max \left\{P_{i}\left(z, \nu_{i}\right) \mid z \in X_{i}\right\}\right.$ the best response correspondence of player $i$ given belief $\nu_{i}$. The set $R_{i}\left(\nu_{i}\right)$ is well defined and compact by Lemma 4 .

We say that an idempotent measure $\nu \in I X$ is supported on a closed set $A \subset X$ if for each continuous function $\varphi: X \rightarrow[0, \infty)$ such that $\varphi \mid A \equiv 0$ we have $\nu(\varphi)=0$.

Lemma 5. Let $A_{i}$ be a closed subset of a compactum $X_{i}$ and $\nu_{i} \in I X_{i}$ such that $\nu_{i}$ is supported on $A_{i}$ for each $i \in\{1, \ldots, n\}$. Then $\otimes_{i=1}^{n} \mu_{i}$ is supported on $\prod_{i=1}^{n} A_{i}$.

Proof. Consider the case $n=2$. Consider any continuous function $\varphi: X_{1} \times X_{2} \rightarrow[0, \infty)$ such that $\varphi \mid A_{1} \times A_{2} \equiv 0$. For $n \in \mathbb{N}$ put

Each $O_{n}$ is an open set in $X_{1} \times X_{2}$ and

$$
O_{n}=\left\{(x, y) \in X_{1} \times X_{2} \mid \varphi(x, y)<1 / n\right\} .
$$

$$
A_{1} \times A_{2} \subset O_{n+1} \subset \mathrm{ClO}_{n+1} \subset O_{n}
$$

for each $n \in \mathbb{N}$. 
Since $A_{1}$ and $A_{2}$ are compact, using induction we can choose for each $n \in \mathbb{N}$ open sets $U_{n} \subset X_{1}$ and $V_{n} \subset X_{2}$ such that $A_{1} \times A_{2} \subset U_{n} \times V_{n} \subset O_{n}, \mathrm{Cl} U_{n+1} \subset U_{n}$ and $\mathrm{Cl} V_{n+1} \subset V_{n}$. Using induction we can also build continuous functions $\psi_{1}: X_{1} \rightarrow[0, \infty)$ and $\psi_{2}: X_{2} \rightarrow[0, \infty)$ such that $\psi_{1}\left|A_{1} \equiv 0, \psi_{2}\right| A_{2} \equiv 0$, $\psi_{1}\left|\left(X_{1} \backslash U_{n}\right) \geq 1 / n-1, \psi_{2}\right|\left(X_{2} \backslash V_{n}\right) \geq 1 / n-1$ for each $n \geq 2, \psi_{1} \mid\left(X_{1} \backslash U_{1}\right) \geq \max \varphi$ and $\psi_{2} \mid\left(X_{2} \backslash V_{1}\right) \geq \max \varphi$.

Since $\nu_{1}$ is supported on $A_{1}$ and $\nu_{2}$ is supported on $A_{2}$, we have $\nu\left(\psi_{1}\right)=\nu\left(\psi_{2}\right)=0$. It follows from the properties of tensor product that $\nu_{1} \otimes \nu_{2}\left(\psi_{1} \circ p_{1}\right)=\nu_{1} \otimes \nu_{2}\left(\psi_{2} \circ p_{2}\right)=0$, where $p_{1}: X_{1} \times X_{2} \rightarrow X_{1}$ and $p_{2}: X_{1} \times X_{2} \rightarrow X_{2}$ are the natural projections. But we have $\varphi \leq \psi_{1} \circ p_{1} \oplus \psi_{2} \circ p_{2}$. Since $\nu_{1} \otimes \nu_{2}$ preserves $\oplus$, we obtain $\nu_{1} \otimes \nu_{2}(\varphi)=0$.

The general case can be obtained by induction.

Definition 2. A belief system $\left(\nu_{1}, \ldots, \nu_{n}\right)$, where $\nu_{i} \in I\left(X_{-i}\right)$, is called a strategic idempotent equilibrium if each $\nu_{i}$ is supported on $\prod_{j \neq i} R_{j}\left(\nu_{j}\right)$.

The main goal of this section is to prove the existence of strategic idempotent equilibrium, moreover we will show that each belief can be represented as a tensor product of corresponding idempotent measures on $X_{i}$. We will need a counterpart of fixed point theorem for Max-Plus convexity.

By a multimap (set-valued map) of a set $X$ into a set $Y$ we mean a map $F: X \rightarrow 2^{Y}$. We use the notation $F: X \multimap Y$. If $X$ and $Y$ are topological spaces, then a multimap $F: X \multimap Y$ is called upper semi-continuous (USC) provided for each open set $O \subset Y$ the set $\{x \in X \mid F(x) \subset O\}$ is open in $X$. It is well-known that a multimap between compacta $X$ and $Y$ with closed values is USC iff its graph is closed in $X \times Y$.

Let $F: X \multimap X$ be a multimap. We say that a point $x \in X$ is a fixed point of $F$ if $x \in F(x)$. The following counterpart of Kakutani theorem for an abstract convexity is a partial case of Theorem 3 from [18] (it also could be obtain combining Theorem 6.15, Ch.IV and Theorem 4.10, Ch.III from [16]).

Theorem 4. Let $\mathcal{C}$ be a normal convexity on a compactum $X$ such that all convex sets are connected and $F: X \multimap X$ is a USC multimap with values in $\mathcal{C} \backslash\{\emptyset\}$. Then $F$ has a fixed point.

Now we are able to prove the main theorem.

Theorem 5. There exists $\left(\mu_{1}, \ldots, \mu_{n}\right) \in I\left(X_{1}\right) \times \cdots \times I\left(X_{n}\right)$ such that $\left(\mu_{1}^{*}, \ldots, \mu_{n}^{*}\right)$ is a strategic idempotent equilibrium, where $\mu_{i}^{*}=\otimes_{j \neq i} \mu_{j}$.

Proof. For each $i \in\{1, \ldots, n\}$ consider a multimap $\gamma_{i}: \prod_{j=1}^{n} I\left(X_{j}\right) \multimap I\left(X_{i}\right)$ defined as follows

$$
\gamma_{i}\left(\mu_{1}, \ldots \mu_{n}\right)=\left\{\mu \in I\left(X_{i}\right) \mid \mu \text { is supported on } R_{i}\left(\mu_{i}^{*}\right)\right\} \text {. }
$$

Put $\mathcal{K}_{i}\left(\mu_{i}^{*}\right)=\left\{\varphi \in C\left(X_{i}\right) \mid \varphi \geq 0\right.$ and $\left.\varphi \mid R_{i}\left(\mu_{i}^{*}\right) \equiv 0\right\}$. Then we have

$$
\left\{\mu \in I\left(X_{i}\right) \mid \mu \text { is supported on } R_{i}\left(\mu_{i}^{*}\right)\right\}=\bigcap_{\varphi \in \mathcal{K}_{i}\left(\mu_{i}^{*}\right)}\left\{\mu \in I\left(X_{i}\right) \mid \mu(\varphi)=0\right\} \text {. }
$$

It follows from the definition of topology on $I\left(X_{i}\right)$ that the set $\left\{\mu \in I\left(X_{i}\right) \mid \mu(\varphi)=0\right\}$ is closed in $I\left(X_{i}\right)$. Hence $\gamma_{i}\left(\mu_{1}, \ldots \mu_{n}\right)$ is a closed subset of $I\left(X_{i}\right)$ for each $\left(\mu_{1}, \ldots \mu_{n}\right) \in \prod_{j=1}^{n} I\left(X_{j}\right)$.

Consider any $\mu, \nu \in I\left(X_{i}\right)$ and $\varphi \in \mathcal{K}_{i}\left(\mu_{i}^{*}\right)$ such that $\mu(\varphi)=\nu(\varphi)=0$. Then we have $\alpha \odot \mu \oplus \beta \odot \nu(\varphi)=0$ for all $\alpha, \beta \in \mathbb{R}_{\max }$ with $\alpha \oplus \beta=0$. Hence $\gamma_{i}\left(\mu_{1}, \ldots \mu_{n}\right)$ is a Max-Plus convex subset of $I\left(X_{i}\right)$ for each $\left(\mu_{1}, \ldots \mu_{n}\right) \in \prod_{j=1}^{n} I\left(X_{j}\right)$.

Define a multimap $\gamma: \prod_{j=1}^{n} I\left(X_{j}\right) \multimap \prod_{j=1}^{n} I\left(X_{j}\right)$ by the formula $\gamma\left(\mu_{1}, \ldots, \mu_{n}\right)=\prod_{i=1}^{n} \gamma_{i}\left(\mu_{1}, \ldots, \mu_{n}\right)$. Let us show that $\gamma$ is USC. Consider any pair $(\mu, \nu) \in \prod_{j=1}^{n} I\left(X_{j}\right) \times \prod_{j=1}^{n} I\left(X_{j}\right)$ such that $\nu \notin \gamma(\mu)$. Then there exists $i \in\{1, \ldots, n\}$ and a function $\varphi \in \mathcal{K}_{i}\left(\mu_{i}^{*}\right)$ such that $\nu_{i}(\varphi)=a>0$. Put $B=\left\{x \in X_{i} \mid \varphi(x) \leq a / 2\right\}$. Evidently $R_{i}\left(\mu_{i}^{*}\right) \subset \operatorname{Int} B$. We can choose a continuous function $\psi: X_{i} \rightarrow[0,+\infty)$ such that $\psi \mid B \equiv 0$ and $\|\varphi-\psi\| \leq a / 2$. Since $\nu$ is non-expanding, we have $\nu_{i}(\psi) \geq a / 2$. Put $O_{\nu}=\left\{\beta \in \prod_{j=1}^{n} I\left(X_{j}\right) \mid \beta_{i}(\psi)>0\right\}$. Then $O_{\nu}$ is an open neighborhood of $\nu$. It follows from Lemma 4 and continuity of tensor product that there exists 
an open neighborhood $O_{\mu}$ of $\mu$ such that for each $\alpha \in O_{\mu}$ we have $R_{i}\left(\alpha_{i}^{*}\right) \subset B$. Hence for each $(\alpha, \beta) \in O_{\mu} \times O_{\nu}$ we have $\beta \notin \gamma(\alpha)$ and $\gamma$ is USC.

We consider on $\prod_{j=1}^{n} I\left(X_{j}\right)$ the family $\mathcal{C}=\left\{\prod_{i=1}^{n} C_{i} \mid\right.$ each $C_{i}$ is Max-Plus convex closed subset of $\left.I\left(X_{i}\right)\right\}$. It is easy to see that $\mathcal{C}$ forms a normal convexity on a compactum $\prod_{j=1}^{n} I\left(X_{j}\right)$ such that all convex sets are connected. Then by Theorem 4 the multimap $\gamma$ has a fixed point $\mu=\left(\mu_{1}, \ldots \mu_{n}\right) \in \prod_{j=1}^{n} I\left(X_{j}\right)$. Let us show that $\left(\mu_{1}^{*}, \ldots, \mu_{n}^{*}\right)$ is an equilibrium under uncertainty. Consider any $i \in\{1, \ldots, n\}$. Then $\mu_{i}$ is supported on $R_{i}\left(\mu_{i}^{*}\right)$. We have by Lemma 5 that $\mu_{i}^{*}$ is supported on $\prod_{j \neq i} R_{j}\left(\mu_{j}^{*}\right)$.

Finally we consider an example which illustrates a difference between Nash equilibrium and strategic idempotent equilibrium. Consider a two-players game $p:\{0,1\} \times\{0,1\} \rightarrow \mathbb{R}$, where the payoff functions $p_{1}$ and $p_{2}$ are defined as follows:

and

$$
p_{1}(0,0)=-1, p_{1}(0,1)=1 / 2, p_{1}(1,0)=1, p_{1}(1,1)=-1
$$

$$
p_{2}(0,0)=1, p_{2}(0,1)=-1, p_{2}(1,0)=-1, p_{2}(1,1)=1 \text {. }
$$

Denote by $\nu_{0}$ the maximal element of $I\{0,1\}$ defined by $\nu_{0}(\psi)=\max \{\psi(x) \mid x \in 0,1\}$ for $\psi \in C(0,1)$. Then the pair $\left(\nu_{0}, \nu_{0}\right)$ is Nash max-equilibrium for the game with expected payoff functions $e u_{i}: I\{0,1\} \times I\{0,1\} \rightarrow \mathbb{R}$, described in Section 3.

The set $\{0,1\}$ is the minimal set, which supports $\nu_{0}$. But $0 \notin R_{1}\left(\left(\nu_{0}\right)^{*}\right)$. Hence $\left(\nu_{0}, \nu_{0}\right)$ is not a strategic idempotent equilibrium.

\section{REFERENCES}

[1] C.D.Aliprantis, M.Florencano, R.Tourky General equilibrium analisis in ordered topological vector spaces, J. Math. Econom. 40 (2004) 247-269.

[2] W.Briec, Ch.Horvath Nash points, Ku Fan inequality and equilibria of abstract economies in Max-Plus and $\mathbb{B}$-convexity, J. Math. Anal. Appl. 341 (2008), 188-199.

[3] W.Briec, Ch.Horvath, A.Rubinov Separation in B-convexity, Pac. J. Optim. 1 (2005), no. 1, 13 Ű30.

[4] J.Dow, S.Werlang, Nash equilibrium under Knightian uncertainty: breaking down backward induction,J Econ. Theory 64 (1994) 205-224.

[5] J.Eichberger, D.Kelsey, Non-additive beliefs and strategic equilibria, Games Econ Behav 30 (2000) 183-215.

[6] I.Gilboa, Expected utility with purely subjective non-additive probabilities, J. of Mathematical Economics 16 (1987) 65-88.

[7] D.Glycopantis, A.Muir, Nash equilibria with Knightian uncertainty; the case of capacities, Econ. Theory 37 (2008) $147-159$.

[8] R.Kozhan, M.Zarichnyi, Nash equilibria for games in capacities, Econ. Theory 35 (2008) 321-331.

[9] Q.Luo, KKM and Nash equilibria type theorems in topological ordered spaces, J. Math. Anal. Appl. 264 (2001) 262-269.

[10] V.P. Maslov, S.N. Samborskii, Idempotent Analysis, Adv. Soviet Math., vol. 13, Amer. Math. Soc., Providence, 1992.

[11] T.Radul, Equilibrium under uncertainty with Sugeno payoff, arXiv:1503.00563

[12] T.Radul, Nash equilibrium for binary convexities, Topological Methods in Nonlinear Analisis 48 (2016), 555-565.

[13] T.Radul, Max-Plus convex compacta: a categorical approach, International conference dedicated to the 120th anniversary of Kazimierz Kuratowski. Lviv, Ukraine, September 27- October 1, 2016. P.44

[14] D.Schmeidler, Subjective probability and expected utility without additivity, Econometrica 57 (1989) 571-587.

[15] Swirszcz T., Monadic functors and convexity, Bull. Pol. Acad. Sci., 22 (1970), 39-42.

[16] M.van de Vel, Theory of convex strutures, North-Holland, 1993.

[17] X. Vives, Nash equilibrium with strategic complementarities, J. Math. Econom. 19 (1990) 305-321.

[18] A.Wieczorek The Kakutani property and the fixed point property of topological spaces with abstract convexity, J. Math. Anal. Appl. 168 (1992), 483-499.

[19] M.Zarichnyi Spaces and mappings of idempotent measures, Izv. Ross. Akad. Nauk Ser. Mat., 74 (2010), 45-64.

[20] K. Zimmermann, A general separation theorem in extremal algebras, Ekon.-Mat. Obz. 13 (1977) 179-201. 